\title{
Specify Number of Lymph Nodes Positive for Tumor by IHC Staining Only
}

National Cancer Institute

\section{Source}

National Cancer Institute. Specify Number of Lymph Nodes Positive for Tumor by IHC

Staining Only. NCl Thesaurus. Code C159765.

A directive to specify the number of lymph nodes found positive for tumor by immunohistochemical (IHC) staining procedures. 